\title{
Rotman-Lens as a True-Time-Delay Beamformer at Low UHF Frequencies
}

\author{
A. Lambrecht ${ }^{(1)}$, T. Zwick ${ }^{(1)}$, Senior, W. Wiesbeck ${ }^{(1)}$, Fellow, \\ J. Schmitz ${ }^{(2)}$, M. Jung ${ }^{(2)}$ \\ (1) Institut für Höchsfrequenztechnik und Elektronik, Universität Karlsruhe (TH), \\ Engesserstraße 5, 76131 Karlsruhe, Germany, \\ http://www.ihe.uni-karlsruhe.de
}

(2) Rheinmetall Waffe Munition GmbH, Heinrich-Ehrhardt-Str. 2, 29345

Unterlüß, Germany

\section{Introduction}

As a well-known broadband device with simple fabrication processes the Rotman lens is applied in various fields. Because it substitutes a whole "switched line length" concept in one passive structure it is investigated here for a true-timedelay beamformer in the frequency range of $450 \mathrm{MHz}$ to $1000 \mathrm{MHz}$. The problem of the Rotman lens is, that it is a traveling wave structure, which has to be sized in the order of several wavelengths to give a proper functionality. This leads to hard requirements considering the size of the Rotman-lens, which will be determined mainly by cost and size of the available substrate. The design of Rotman lenses is hard at lower frequencies and this is the first time this structure was investigated for the low UHF band.

In general there exist various methods to steer a beam in an antenna array [1]. To get the proper phase distribution along the used dimension of the array phase shifters are needed. Normally in narrow-band systems these are ferrite phase shifters or PIN-diode phase shifters, i.e. constant-phase-type (CPT) phase shifter. The problem forming a proper wave front in the desired direction becomes severe, when the bandwidth and the steering angle grow and true-time-delay (TTD) phase shifters are needed. The CPT only works monochromatically or narrow-band. So for the phase front of the feeding there is only one pair of frequency and steering angle $f_{0}, \theta_{0}$ where it is consistent with the phase front of the radiated field. For another frequency and angles the following condition holds:

$$
\left(f_{0}+\Delta f\right) \cdot \sin \left(\theta_{0}+\Delta \theta\right)=f_{0} \sin \left(\theta_{0}\right)
$$

So for a change in frequency we get for the change in angle:

$$
\Delta \theta=\arcsin (\psi)-\left.\theta_{0}\right|_{\psi=\frac{f_{0}}{f_{0}+\Delta f} \cdot \sin \left(\theta_{0}\right)}
$$

By taking the middle frequency as $f_{0}$ and the maximum deviation at the end of the band $\Delta f=275 \mathrm{MHz}$ an angle error of $\Delta \theta=-14.16^{\circ}$ occurs.

A TTD phase shifter does not fix the phase but, of course, the traveling time of the electromagnetic wave:

$$
\tau_{i}=\frac{1}{c_{0}}\left(\frac{N+1}{2}-i\right) d \sin \left(\theta_{0}\right)
$$


This equation assumes an antenna array with spacing of $d$ and $N$ elements. $\tau_{i}$ is the delay for the i-th element of the array, the reference point is the middle of the array. This leads to frequency dependent phasing of the array, compensating the frequency change of the traveling wave, leading to a stationary far-field solution. There exist various methods to realize a true-time-delay beamforming network, i.e. switching of transmission lines (microstrip, coaxial, waveguide), switching of optical lines, use of traveling wave structures (Rotman lens, other lenses).

The system concept comprises a 1 x 4 phased array antenna, for which the Rotman lens provides the ultra-wideband phasing of the antenna feeds. The lens is fed by SP9T PIN-switch, which provides the different scan angles positions.

The system specifications define a scan angle range of $\pm 40^{\circ}$, a frequency range of $450 \mathrm{MHz}-1000 \mathrm{MHz}$ (bandwidth of 75\%), desired VSWR of 2:1 and the number of beam directions is 9 . The system will be initially used in CW mode.

\section{Realization of the Beamformer with a Rotman-Lens Design}

The basic design equations for Rotman Lenses can be found in [2], [3]. These are based on ray-optical modeling of the parallel plate waveguide with feeding points on the arrayport and beamport contour [4]. There also exist some design rules for the optimum port layout, when using microstrip technology [5]. A numerical field solver is used to calculate the Rotman-Lens structure.

The prototype for the frequency range of $450 \mathrm{MHz}$ to $1000 \mathrm{MHz}$ is etched on an Arlon substrate with a dielectric constant of 10.9 and dimensions of $609.6 \mathrm{~mm} \mathrm{x}$ $914.4 \mathrm{~mm} \times 3.23 \mathrm{~mm}$. In Fig. 1 the prototype is shown. So the available space is only $3 \lambda_{450 \mathrm{MHz}}$ in the wave traveling direction ( $\mathrm{x}$-dimension), because the $\mathrm{y}$ dimension $(914.4 \mathrm{~mm}$ ) has to be used for designing the dummyport region of the lens. Often Rotman-Lenses have extensively formed dummy regions; geometrical reasons restrict that here. The Fig. 2 shows the surface currents for excitation of $\mathrm{BP}_{1}$ and $\mathrm{BP}_{5}$. For $\mathrm{BP}_{1}$ a non-uniform distribution along the arrayport side is observable. This is partly due to the small $y$-dimensions available for the dummy region, i.e. in terms of wavelengths on the beamport side $1.26 \lambda_{450 \mathrm{MHz}}$ / $2.81 \lambda_{1000 \mathrm{MHz}}$ and on the arrayport side $0.88 \lambda_{450 \mathrm{MHz}} / 1.95 \lambda_{1000 \mathrm{MHz}}$. The structure has 14 dummyports on the sidewall-region and 3 dummyports between the arrayports.

The lens without absorber attached to the dummyport region shows a sufficient matching of the beamports only from $600 \mathrm{MHz}$ onwards; with the absorber (dark parts) the matching is better, approximately from $570 \mathrm{MHz}$ onwards. The used absorber material is C-RAM FDSS ${ }^{\circledR}$. An excellent phase behavior in the whole frequency range is achieved (see Fig. 4). The gain patterns show the stationary beams for $\mathrm{BP}_{1}$ and $\mathrm{BP}_{5}$. These gain patterns were synthesized by using measurement results. The grating lobe for higher frequencies visible in Fig. 4 left for $\mathrm{BP}_{1}$ is due to the pre-defined antenna array spacing of $30 \mathrm{~cm}$, corresponding to the free-space wavelength at $1 \mathrm{GHz}$. In these gain patterns a measured Vivaldi antenna with a frequency dependent gain is used as single element for the array. This and the transmission variance of the Rotman-Lens lead to the gain deviation visible in Fig. 4 down left and down right. 


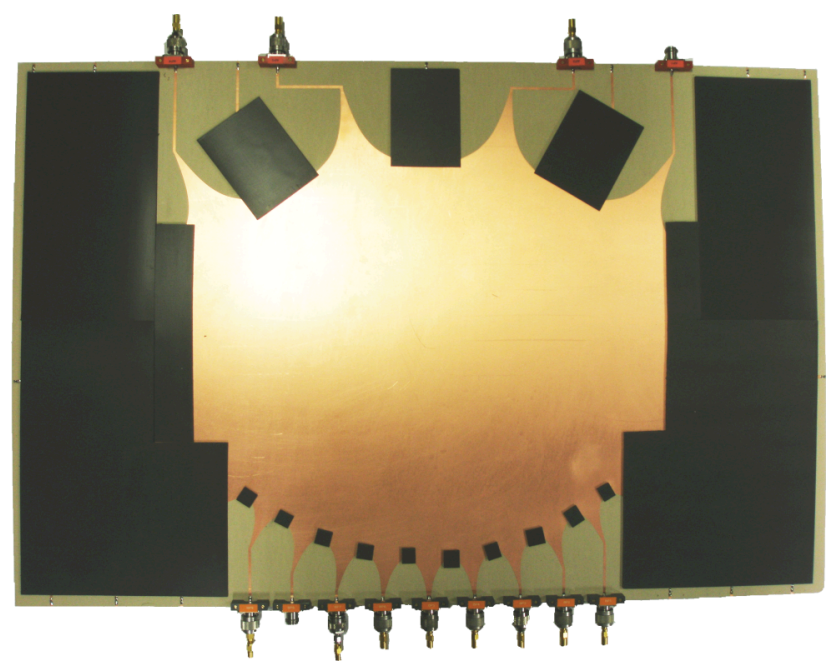

Fig. 1 The prototype Rotman-Lens with N-Connectors and absorbing material (C-RAM FDSS ${ }^{\circledR}$ ) in the dummy region.
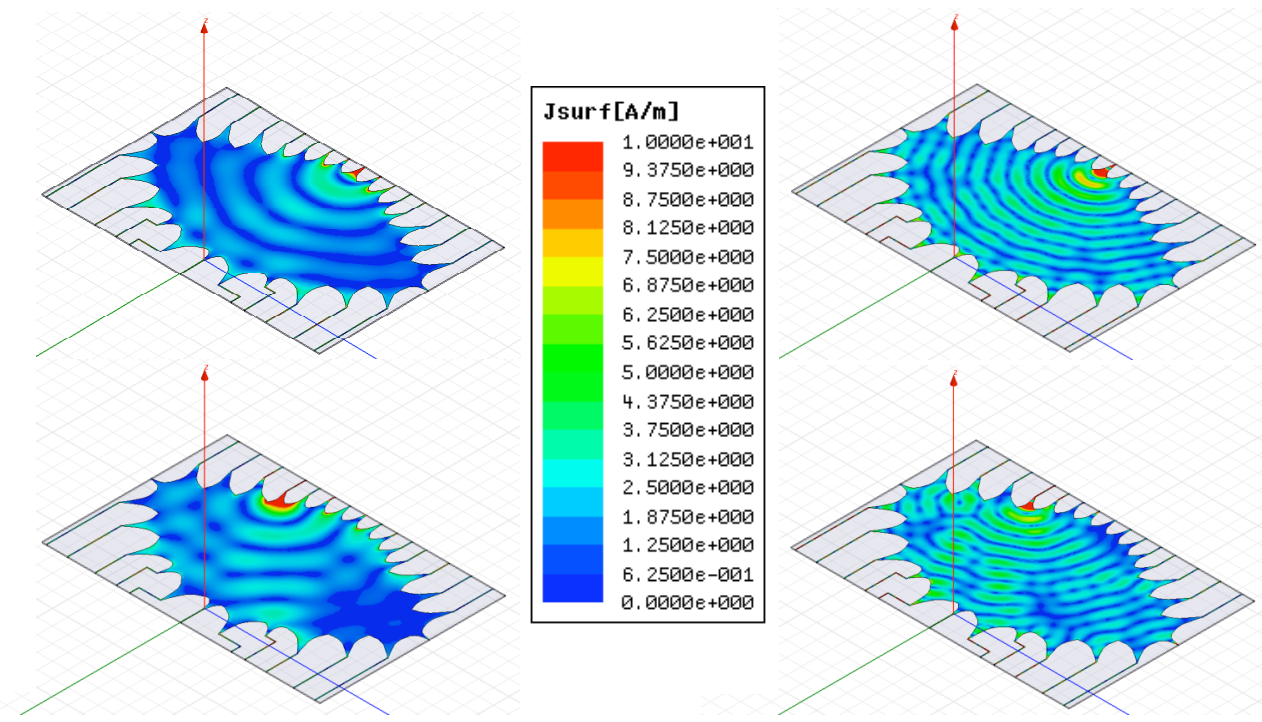

Fig. 2 left: surface currents at $450 \mathrm{MHz}$, right: surface currents at $1000 \mathrm{MHz}$.
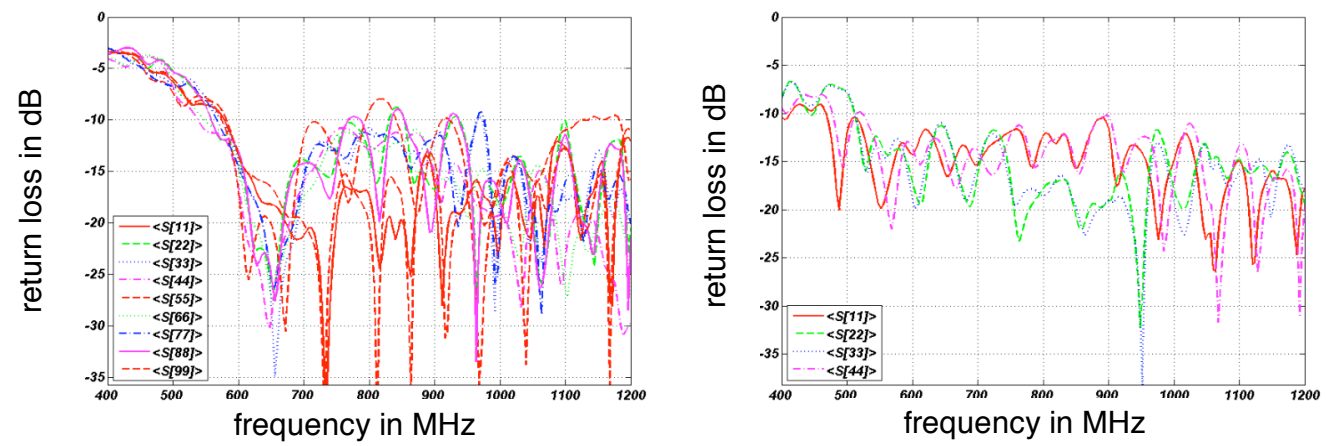

Fig. 3 left: return loss of beamports $\mathrm{BP}_{1}$ to $\mathrm{BP}_{9}$, right: return loss of arrayports $\mathrm{AP}_{1}$ to $\mathrm{AP}_{4}$. 

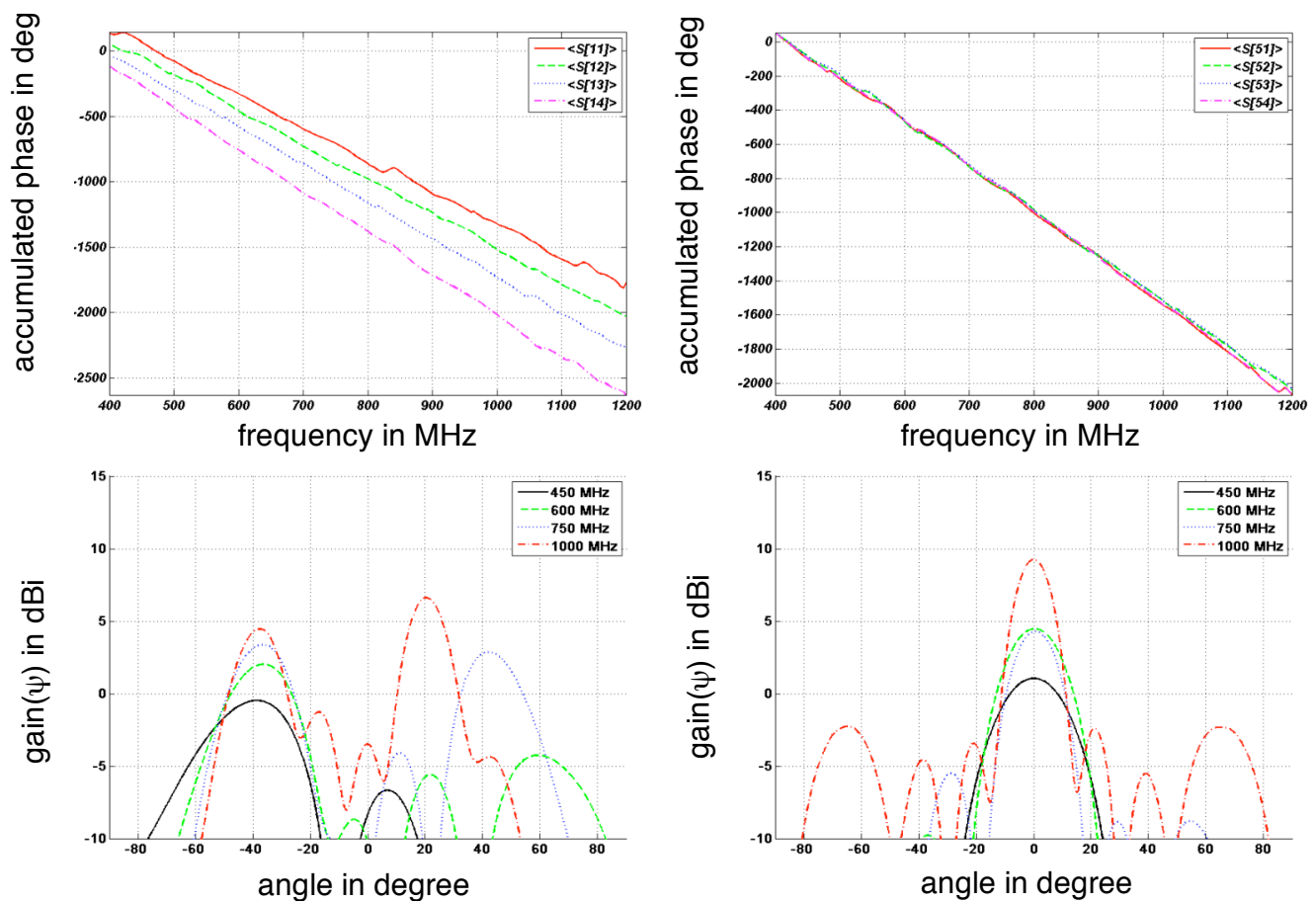

Fig. 4 left: accumulated phase for $\mathrm{BP}_{1}$ and synthesized gain pattern, right: accumulated phase for $\mathrm{BP}_{5}$ and synthesized gain pattern.

If the system operates in pulse-mode the time domain array factor gives the superposition of only 4 pulses from each sending antenna, so no grating lobe will occur. Nonetheless the appearance of grating lobes for $\mathrm{CW}$-mode translates into additional dispersiveness for pulse-mode [6].

\section{Conclusion}

The Rotman-lens was investigated for the first time at low UHF frequencies. The lens structure has a very small dummyport region compared to wavelength. Further research will concentrate on improvements of the matching and hardware implementation into a whole array and pulse-mode operation.

\section{References:}

[1] Johnson, R. C., "Antenna engineering handbook", 3. ed. Norwich, NY : Knovel; New York [u.a.] : McGraw-Hill, 2006.

[2] Rotman W., Turner R.F., "Wide-Angle Microwave Lens for Line Source Applications", IEEE Transactions on Antennas and Propagation, 1963.

[3] Hansen R. C., "Design Trades for Rotman Lenses", IEEE Transactions on Antennas and Propagation, vol. 39, no. 4, April 1991.

[4] Simon P. S., "Analysis and Synthesis of Rotman Lenses", 22nd AIAA International Communications Sattelite Systems Conference \& Exhibit, 2004.

[5] Hall L., Hansen H., Abbott D., "Rotman lens for mm-wavelengths", Proceeding of SPIE, vol. 4935, 2002.

[6] W. Sörgel, C. Sturm, and W. Wiesbeck, "Impulse Responses of Linear UWB Antenna Arrays and the Application to Beam Steering", Proceedings 2005 IEEE International Conference on Ultra-Wideband, pp. 275-281, Zurich, Switzerland, September 2005 